\title{
A cien años de Córdoba: entre imperativos tecno-económicos y la reelaboración de conocimientos
}

\section{A hundred years of Córdoba: between techno-economic imperatives and the re-elaboration of knowledge}

\author{
Alexis Mercado \\ Universidad Central de Venezuela \\ Centro de Estudios del Desarrollo (CENDES) \\ Área de Desarrollo Científico y Tecnológico \\ alexis.mercado@ucv.ve \\ Código Orcid: https://orcid.org/0000-0003-3984-8992
}

\begin{abstract}
Resumen
Un elemento renovador de la Reforma de Córdoba fue el fortalecimiento de la función social de la universidad, dando cabida al desarrollo de su tercera misión (la extensión). Partiendo de ello, se discuten problemas socioambientales globales apremiantes, la situación de la estructura productiva de América Latina y se analizan los elementos más remarcables del desarrollo de las tecnologías disruptivas y la emergencia de la 4i, elementos que tendrán serias implicaciones socioeconómicas para la región. El análisis de la información de diversas fuentes evidencia que a pesar de los avances tecnológicos, prevalecen formas de producción y consumo no sustentables. En América Latina, los problemas se agudizan a consecuencia del predominio de una estructura económica que aún depende de la explotación intensiva de recursos naturales. De no adoptarse acciones adecuadas esta situación empeorará con la irrupción de los nuevos sistemas tecnológicos que demandan los recursos que posee la región. Abordar los problemas socioambientales de manera efectiva requiere reelaborar formas de generación de conocimientos, asumiendo enfoques que trasciendan los modos tradicionales de investigación e incorporando diversos conocimientos y actores sociales. Se propone, entonces, que la extensión, aparte de la proyección cultural, sirva de correaje de intercambio entre las comunidades y los investigadores para generar nuevas agendas y contribuir a la creación de espacios que permitan implantar formas alternativas de realizar la actividad. En esta medida, se transforma también la segunda misión de la universidad.
\end{abstract}

\section{Palabras clave}

Reforma, Córdoba, crisis socio-ambiental, extensión, investigación.

Forma sugerida de citar: Mercado, Alexis (2018). A cien años de Córdoba: entre imperativos tecno-económicos y la reelaboración de conocimientos. Universitas, 29, pp. 67-87. 


\begin{abstract}
The Reform of Cordoba reinforced the social function of the university, allowing the development of its third mission (the Extension). In this article, urgent global socio-environmental problems and the situation of the productive structure of Latin America are discussed, and the most outstanding elements of the development of disruptive technologies and the emergence of the $4 i$ are analyzed. All of them will have serious socio-economic implications for the region. The analysis of information from different sources shows that despite technological advances, unsustainable forms of production and consumption prevail. In our region, these problems are exacerbated by the predominance of an economic structure that still depends on the intensive exploitation of natural resources, which, if appropriate measures are not adopted, will worsen with the irruption of the new technological systems. This because demand natural resources that abound in the region. An effective approach of socio-environmental problems requires the re-elaboration of forms of generation of knowledge, assuming approaches that overcome the traditional ways of research incorporating diverse knowledge and social actors. It is proposed that the extension works as a conveyor belt for exchange information between communities and researchers to generate new agendas and contribute to develop spaces that allow the implementation of alternative ways of carrying out the activity. To this extent, the second mission of the university is also transformed
\end{abstract}

Keywords

Córdoba reform, socio-environmental crisis, extension, research.

\title{
Introducción
}

La reforma de Córdoba, aparte de impulsar transformaciones en la universidad que apuntaban a la autonomía y la democratización de sus formas de gobierno, y desde el punto de vista académico a la libertad de cátedra y cambios en la organización y los métodos de enseñanza, supuso también la transformación de sus relaciones con la sociedad, proponiendo un fortalecimiento de su función social mediante la que, a la postre, se constituyó en su tercera misión (la extensión), y una mayor preocupación por los problemas nacionales (Tünnermann, 2008).

A cien años de este hito histórico de la educación superior latinoamericana, la universidad es blanco de cuestionamientos que en su mayoría se relacionan justamente con sus escasos aportes a la sociedad en temas que van desde su baja contribución al desarrollo económico-productivo al escaso abordaje de apremiantes problemas como la exclusión social y la degradación ambiental. 
Los reclamos en uno u otro sentido reflejan una controversia acerca de la naturaleza y función que debe tener la universidad en la que se confrontan, con diversos matices, dos posiciones antagónicas: la que le considera como bien público y social que debe contarse entre las prioridades del Estado (Hitner et al., 2017) y la que le concibe como un commodity privado (Williams, 2016) internacionalmente comercializable a un grado tal que ha llegado a discutirse en el seno del Acuerdo General Sobre Comercio y Servicios (AGCS) como un tema de su competencia.

Es evidente que las orientaciones que adopten las IES (Instituciones de Educación Superior) tendrán profundas implicaciones sobre la generación, transmisión y apropiabilidad de los conocimientos. Es incuestionable que las IES tienen la obligación de contribuir al desarrollo económico de sus países, más aún cuando se admite que avanzamos hacia una economía basada en el conocimiento. Pero esta constituye una entre varias prioridades y no - como se pretende evidenciar en el modelo de universidad emprendedora - la principal. Tan o más urgentes resulta el abordaje de la participación social y los impactos socioambientales derivados de las actividades antrópicas.

Se reconoce que transcurrido un siglo de la reforma de Córdoba, la universidad latinoamericana ha desempeñado un rol determinante en el desarrollo social y cultural de sus países. La ampliación del acceso a la educación superior ha contribuido a la inclusión social, a la vez que ha permitido la conformación de importantes comunidades profesionales en las diferentes áreas del conocimiento, en especial las tradicionales y - en menor medida - en las científicas y tecnológicas. Pero de manera general, no ha logrado acompañar los avances científicos y tecnológicos internacionales (Vessuri, 2004), contribuyendo muy poco tanto para la transformación de una estructura económica que presenta una matriz productiva fuertemente basada en actividades primarias y marcadas condiciones de dependencia como para proponer soluciones a importantes problemas socioambientales.

Tal situación presiona para una revisión de sus vínculos con la sociedad. Se le demanda que sus funciones sobrepasen los límites disciplinarios en los procesos de enseñanza- aprendizaje y la investigación, para lo que, imperativamente, debe trascender sus "muros" involucrándose activamente en el estudio y abordaje de problemas sociales. Sin embargo, a ello se interpone su lentitud para adaptarse, tanto estructural como funcionalmente, a los acelerados cambios que se experimentan en los diversos ámbitos de la sociedad (Peñalver en Hitner et al., 2017). Entre estos, destacan dos para las IES: la acelera- 
ción del desarrollo tecno-científico - manifestado en el auge de una serie de tecnologías disruptivas y la emergencia de la cuarta revolución industrial - y recientes movimientos de reelaboración de las formas de producción de conocimiento que promueven una mayor participación de las comunidades, la interculturalidad y el diálogo de saberes (Hitner et al., 2017). La ineludible incorporación de ambas en sus agendas dependerá, en gran medida, de la disposición para introducir cambios sustanciales en su naturaleza y funciones.

Este artículo destaca brevemente las orientaciones de la vinculación con la sociedad que planteó la Reforma de Córdoba, las cuales sirven de fondo para presentar una discusión sobre los problemas socioambientales globales más acuciantes y la situación de la estructura productiva de la región, que hacen imperativo su consideración por parte de las IES. Posteriormente, se analizan los elementos más resaltantes del desarrollo de las tecnologías disruptivas y la emergencia de la cuarta revolución industrial — que tendrán implicaciones socioeconómicas muy importantes - , y de la reelaboración de las formas de producción de conocimiento que presionan una revisión de los significados y alcances de las funciones de estas instituciones. Como colofón, se sugieren cambios en la orientación de las misiones de extensión e investigación para afrontar los ineludibles desafíos tecno-económicos y de nuevas formas de producción de conocimiento.

\section{La preocupación social en la Reforma}

La Reforma de Córdoba supuso, antes que todo, una transformación interna de la universidad. Partiendo del cuestionamiento al modelo universitario tradicional, planteó la obligación de llevar los conocimientos al pueblo. Contribuir al incipiente proceso de cambio social que comenzaba a experimentar la región fue el estímulo fundamental que estableció las pautas de la vinculación de la universidad con la sociedad (Anuies, 1979 en Serna Alcántara, 2007).

En términos de interacción e integración social, la influencia más trascendente de las ideas de la Reforma fue abrir las posibilidades de acceso a los jóvenes de estratos sociales menos favorecidos: "A los hijos de las capas sociales emergentes" (Tünnermann, 2008), ocurrida sobre todo durante la década de los sesenta, al compás de los hechos políticos vividos en la región. Pero este autor destaca que en el Manifiesto liminar de la Reforma se proclama la misión social de la universidad y la extensión universitaria, 
cuya función sería "la proyección al pueblo de la cultura universitaria y la preocupación por los problemas nacionales" (Tünnermann, 2008).

Esta perspectiva se arraigó fuertemente en estas instituciones. La extensión como medio de proyección (unidireccional) es la visión que ha prevalecido, concibiéndola como un mecanismo de socialización de la cultura que frecuentemente se limita a acciones de difusión cultural y actividades extra escolares, y la provisión de asistencia social (Fresan, 2004). Esta situación cuestionaría la validez del principio de indisociabilidad enseñanza-investigación-extensión (Baptista y Kuenzer en Fresán Orozco, 2004), creando obstáculos para una mayor inserción de la universidad en el tejido social.

En la actualidad, los problemas socioambientales y el imperativo de orientar las sociedades hacia trayectorias más sustentables, plantean un reenfoque de la vinculación y una renovación de la extensión que tendrán consecuencias sobre la formación y la investigación. La universidad debe superar las estrategias de socialización, promoviendo una participación ciudadana más activa que sirva de correaje para intercambiar información y conocimiento que ayude a enfocar estos problemas y contribuir conjuntamente a su resolución.

\section{Crisis socioambiental}

El trienio 2015-2017 registró las temperaturas más altas de la historia (WMO, 2018). Los escenarios del calentamiento global proyectados por el IPCC para 2100 prevén que aun disminuyendo el crecimiento de las emisiones de gases efecto invernadero (GEI) de acuerdo a los compromisos del acuerdo de París (2015), este no será inferior a $2{ }^{\circ} \mathrm{C}$. Los rangos probables de incremento lo ubican entre 2 y $4,9^{\circ} \mathrm{C}$, con una media de $3,2^{\circ} \mathrm{C}$ y con apenas $5 \%$ de probabilidad que sea menor de $2{ }^{\circ} \mathrm{C}$. El problema radica en el incremento de la cantidad de GEI en la atmósfera proveniente de actividades antrópicas (WMO, 2017). ${ }^{1}$

El aumento de la temperatura acrecienta los eventos ambientales extremos. Está ocasionando un aumento en el nivel de los océanos y su acidificación, lo cual afecta a la biodiversidad. El aumento de la frecuencia de fuertes precipitaciones, el incremento neto de la pluviosidad, así como de la

1 En 2016 la concentración de CO2 alcanzó records históricos, ubicándose en 403 partes por millón (ppm), 45\% por encima de los niveles preindustriales. En tanto que las de metano llegaron a 1853 ppm (157\% superiores a la concentración de la época preindustrial) y las de óxido nitroso a 329 ppm (22\% superiores) (WMO, 2017). 
frecuencia e intensidad de huracanes tropicales, son efectos constatables del cambio climático. También se evidencia una intensificación de las sequías y aumentos en la desertificación y desaparición de glaciares (IPCC, 2012). Tal situación coloca en extrema vulnerabilidad a centenares de millones de habitantes del planeta y la mayoría de los países de América Latina y el Caribe son sensiblemente vulnerables a estos eventos.

Por su parte, el crecimiento de la explotación de recursos naturales y el incremento de la producción industrial generan nuevas sustancias xenobióticas que agravan el problema de la contaminación y la afectación de la salud de los seres vivos. Por ejemplo, diariamente se descargan dos millones de toneladas de aguas residuales y desechos industriales y agrícolas en los cursos y reservorios de agua del mundo, afectando la disponibilidad de agua potable. Un porcentaje importante de los desechos sólidos presentan baja biodegradabilidad y en algunos casos alta toxicidad. En 2015 se generaron aproximadamente 6300 millones de toneladas de desperdicios plásticos, de los cuales $9 \%$ fueron reciclados, $12 \%$ incinerados y $79 \%$ arrojados en vertederos; de estos, alrededor de 13 millones de toneladas van a parar anualmente a los océanos, produciendo la creciente afectación de la biota marina (Salleh, 2015). Las proyecciones estiman que para 2050 la generación de desechos plásticos alcanzará los 12000 millones de toneladas. Su ubicuidad en el ambiente ha hecho que esta acumulación se sugiera como un indicador geológico de la era del Antropoceno (Geyer et al., 2017).

La principal causa de estos problemas radica en la sostenida expansión de sectores productivos tradicionales y la irrupción de nuevos sistemas tecnológicos ${ }^{2}$ bajo el paradigma del crecimiento continuo, debido a que mantienen -e incluso agravan - el impacto de la explotación y uso de recursos, y la consecuente degradación ambiental por la contaminación. Por ejemplo, la obtención de metales básicos, asociados al paradigma tecno-económico basado en el uso intensivo de materiales y energía, creció aceleradamente en este siglo, incluso por encima de la economía en general. La producción de hierro creció $180 \%$ entre 2002 y 2014 , en tanto que la de aluminio primario $108 \%$. Similarmente, la producción de combustibles fósiles, aunque atenuó su ritmo de crecimiento, se mantiene en escalas extraordinarias. La produc-

2 Se adopta la definición realizada por Hughes (1987) que establece que los sistemas tecnológicos están integrados por componentes técnicos (artefactos y procesos de producción) y organizacionales (empresas manufactureras, de asistencia técnica y financieras). Se integran, además, componentes científicos y de enseñanza, e incluso elementos de legislación como es el caso de normas regulatorias. 
ción de carbón creció $66 \%$, la de petróleo $28 \%$ y la de gas natural $41 \%$. Proporcionalmente, se registra el incremento de las emisiones de GEI, agravando, como se indicó, los problemas del calentamiento global.

A ello se suma que el desarrollo de tecnologías disruptivas estimula la producción de una constelación de nuevos dispositivos y nuevos servicios que ampliarán la demanda de nuevos materiales (los nuevos commodities), especialmente en los países desarrollados, exacerbando la explotación de recursos naturales. Tres elementos químicos (el litio, el niobio y el tántalo) destacan por su funcionalidad para la elaboración de los citados dispositivos, determinándose que la explotación de los materiales naturales que les contienen crece sostenidamente. Entre 2006 y 2014 (apenas ocho años), la producción de litio equivalente aumentó $86 \%$, muy superior al de la economía (52\%), en tanto que la de niobio fue de $76 \%$.

Estos resultados evidencian que lejos de una racionalización de la explotación de recursos inherentes al anterior paradigma tecno-económico, esta se acelera, adicionándose el crecimiento sostenido de la explotación de los nuevos commodities inherentes al nuevo paradigma. De esta manera, puede refutarse uno de los argumentos fundamentales de la tesis de estar avanzando hacia la "economía del conocimiento": el desacoplamiento entre el crecimiento económico y la explotación de recursos. Esto podrá estarse dando en muy contadas economías desarrolladas, pero un balance de masas impide confirmar que se esté llevando a cabo en el ámbito global.

Tanto más paradójico resulta que, a pesar del extraordinario incremento de la explotación de recursos naturales y del sostenido crecimiento de la economía financiera, el mantenimiento o aumento de la exclusión y la pobreza constituyan otros síntomas de la crisis socioambiental. En un reciente informe del Banco Mundial se estimó que la riqueza global - medida como la sumatoria del capital producido, el capital natural, el capital humano y los activos netos en el extranjero- creció 66\% entre 1995 y 2014. El informe señala que esto fue acompañado por una reducción importante de la concentración de la riqueza en los países de alto ingreso. Pero en los de bajo ingreso, que detentan apenas el $1 \%$ de la riqueza global (fundamentada en su patrimonio natural), salvo contadas excepciones, se observaron escasos avances, llegando a producirse una reducción de la riqueza per cápita en un importante número de ellos (WB, 2018).

Vale destacar que las cifras de la riqueza per cápita permiten dimensionar las diferencias globales de la desigualdad. En 2014, en los países de la 
OCDE, una persona al nacer, en promedio, poseía una riqueza equivalente de 708389 dólares, mientras que el promedio de una persona al nacer en un país de bajo ingreso era 13 629,52 veces menor (WB, 2018). Si a ello agregamos las diferencias internas que, como se indicó, son mayores en los países de bajo ingreso, estas brechas se vuelven inconmensurables. Así, resultan mucho más ilustrativos los datos acerca de la riqueza de las ocho personas más adineradas del planeta, riqueza que equivale a los bienes que poseen los 3600 millones más pobres del mundo (Hope, 2017).

\section{América Latina: ¿economía del conocimiento?}

Este panorama global de severa crisis socioambiental se manifiesta claramente en América Latina. Los persistentes niveles de pobreza y exclusión, así como importantes situaciones de deterioro ambiental medida debido a patrones económicos en los que predomina o se ha acentuado la explotación de recursos naturales, resaltan como los temas más acuciantes.

La integración de América Latina a la economía global se da de manera asimétrica, fundamentalmente como proveedor de materia prima, de ahí que los problemas descritos adquieran particular intensidad y el usufructo de los posibles beneficios sea sustancialmente menor. Así, ante la casi imposibilidad de sustraerse de las dinámicas globales de las transformaciones tecnológicas, es imperativa la contribución de sus estructuras científico-técnicas para superar los patrones primarios productivos, condición elemental para avanzar en la transición hacia formas de vida más sustentables.

\section{Perfil económico}

Después del estancamiento experimentado en las dos últimas décadas del siglo XX, América Latina registró índices positivos de crecimiento. Entre 2000 y 2014 la economía se incrementó en 110\% (CEPAL, 2014). ${ }^{3}$ Este período mostró una contracción en 2009 como consecuencia de la crisis global y en los años 2015 y 2016 (-0,4\% y -0,1\% respectivamente) asociada a lo que se ha denominado "el fin del súper ciclo de los commodities".

3 Estimado a precios constantes en U\$ de 2010 (CEPAL). 
El impacto de la caída de los precios de las commodities sobre las economías latinoamericanas refuerza el debate sobre el estancamiento estructural de la región, acentuando la controversia sobre su estructura económica, sostenida cada vez más en los servicios y en actividades primarias, que fundamentan su inserción asimétrica en la economía global. De hecho, la evolución del PIB por tipo de actividad de la región evidencia una alineación con la tendencia global de una creciente participación de los servicios en el PIB, la "dematerialización", pero en muchos casos asociados a la explotación y exportación de recursos naturales.

En el citado periodo, servicios básicos y comercio, transporte, comunicaciones y finanzas, fueron las actividades que registraron el mayor crecimiento. También hay correspondencia en la pérdida de importancia de la manufactura, el renglón que registra la mayor disminución en el crecimiento (tabla 1).

\section{Tabla 1}

\section{PIB anual por actividad económica a precios constantes en dólares*}

\begin{tabular}{|l|l|l|l|}
\hline \multicolumn{1}{|c|}{ Rubro } & \multicolumn{1}{c|}{$\mathbf{1 9 9 0}$} & \multicolumn{1}{c|}{$\mathbf{2 0 1 4}$} & \multicolumn{1}{c|}{ Variación } \\
\hline Agricultura, ganadería, caza, silvicultura y pesca & 136901 & 262613,3 & $91,8 \%$ \\
\hline Explotación de minas y canteras & 187546,8 & 324289,6 & $72,9 \%$ \\
\hline Industrias manufactureras & 437645,3 & 734586,9 & $67,8 \%$ \\
\hline Servicios básicos** & 54052,8 & 132284,6 & $144,7 \%$ \\
\hline Construcción & 178950,8 & 347050,6 & $93,9 \%$ \\
\hline Comercio, transporte, comunicaciones y finanzas & 860992,3 & 2061576,4 & $139,4 \%$ \\
\hline $\begin{array}{l}\text { * Millones de USD } \\
\text { ** Electricidad, gas y agua }\end{array}$ & & \\
\hline
\end{tabular}

Fuente: CEPAL

El PIB de la construcción casi se duplica en el período. Agricultura, ganadería, caza, silvicultura y pesca, actividades dependientes de la biodiversidad, muestran un crecimiento similar, apuntalado en el incremento del agronegocio, que consolidó a Brasil y Argentina, como grandes exportadores de cereales. Finalmente, minas y canteras registró un crecimiento algo inferior, muy ligado a la actividad exportadora.

La variación de la participación de estas actividades en el PIB permite apreciar mejor su peso dentro de la actividad económica. Aunque servicios 
básicos fue la que porcentualmente aumentó más en el período considerado, su participación en el PIB se mantiene marginal (pasa de 2\% a 2,4\%). A ello habría que agregar el extraordinario déficit que presentaban, cuya mejoría y ampliación de cobertura fueron colocadas por diversos organismos internacionales como condición para mejorar la calidad de vida. ${ }^{4}$

\section{Figura 1}

\section{Participación de diferentes actividades económicas en el PIB en América Latina (1990-2015)}

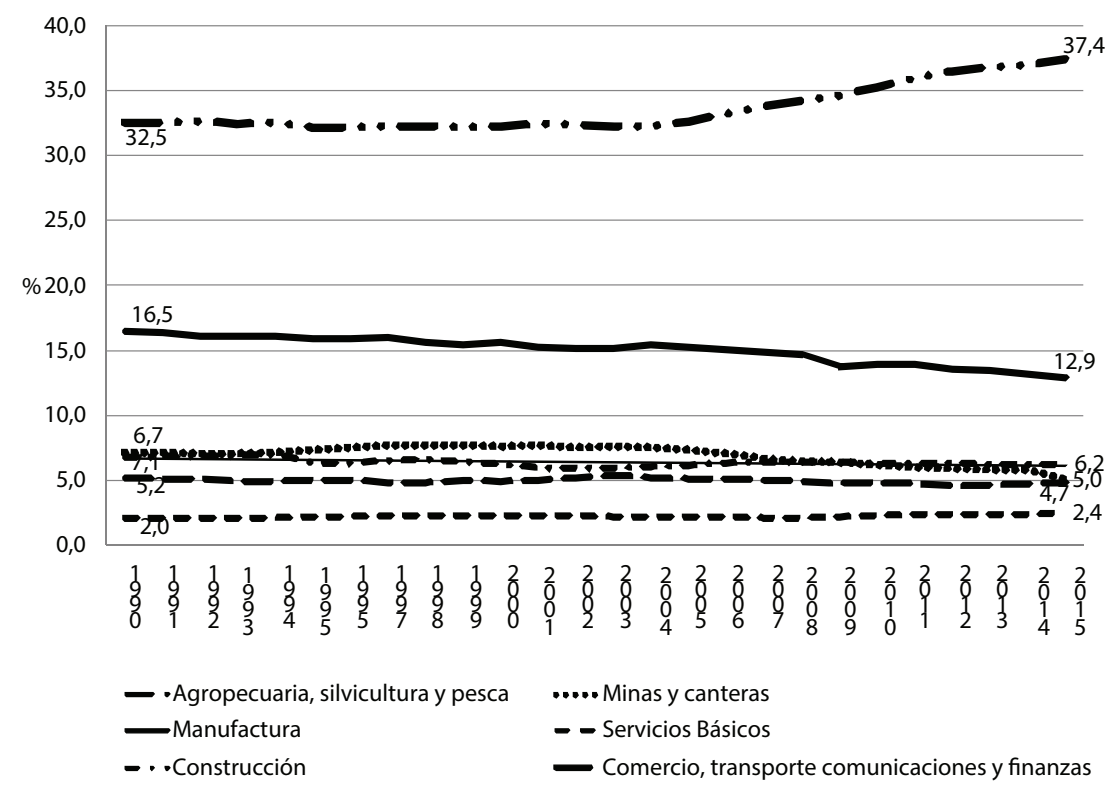

Fuente: CEPAL

Los servicios (comercio, transporte, comunicaciones y finanzas) se consolidan como el sector de más peso en la economía de la región, aumentando

4 A título de ejemplo, las Naciones Unidas establecieron en las Metas del Milenio la necesidad de aumentar la cobertura del acceso al agua mejorada y potable y el saneamiento: para 2015 se debía reducir a la mitad el porcentaje de personas sin acceso sostenible al agua potable y a servicios básicos de saneamiento. 
significativamente su participación de $32,5 \%$ a $37,4 \%$ (incremento de $15 \%$ ), mientras que en el otro extremo se ubica la manufactura cuya participación pasa de $16,5 \%$ a $12,9 \%$ (una disminución de su contribución de $22 \%$ ), inferior al $15 \%$ que presenta la Unión Europea que, consciente de la importancia que tiene esta actividad en el empleo y la generación de bienes, se ha propuesto revertir la tendencia y elevar su participación al 20\% en 2020 (Bussines Europe, 2014). El resto de las actividades, varían poco su participación: construcción (pasa de $6,7 \%$ a $6,2 \%$ ), agricultura $(5,2 \%$ a $4,7 \%)$ y minas y canteras (incluye explotación petrolera, de 7,1\% a 6,2\%) (Figura 1). Aun cuando esta última actividad haya visto ligeramente disminuida su participación en el PIB, el aumento en la explotación de recursos en el presente siglo es significativo. Los tres principales commodities minerales metálicos (hierro, cobre y aluminio-bauxita) muestran un crecimiento sostenido hasta 2008, registrando una caída al año siguiente producto de la crisis económica, pero retomándolo en la década siguiente a pesar de la caída de los precios (Tabla 2).

En 2001, la extracción de mineral de cobre fue de algo más de seis millones de toneladas. Esta creció lenta pero sostenidamente alcanzado 8433000 toneladas en 2015, lo que representa un incremento cercano al $40 \%$. En apenas seis años, la explotación de hierro experimentó un crecimiento de $64 \%$. Después del reflujo de la crisis de 2008, retomó el ritmo de crecimiento alcanzando un máximo de 521 millones de toneladas en 2011, un incremento de $110 \%$ respecto a 2001 . La bauxita también experimentó un alto ritmo de crecimiento entre 2001 y 2008 , cercano al $50 \%$, cayendo de manera importante en 2009 y a partir de 2010 retomó un lento pero sostenido crecimiento de su producción, aproximándose a 53 millones de toneladas en 2015.

\section{Los materiales estratégicos}

Aun cuando no se dispone de datos precisos sobre la explotación de los nuevos commodities y aunque su actual ritmo de explotación no es - todavíaimportante, es probable que se intensifique en los próximos años. Ello porque América Latina posee el 97\% de las reservas mundiales de niobio, 96\% de litio y 54\% de tántalo (Bruckmann, 2015). En otras palabras, detenta el patrimonio de los recursos clave para el desarrollo de los nuevos sistemas tecnológicos.

Es necesario señalar que los procesos de extracción y transformación de estos elementos se basarán, cada vez más, en las tecnologías convergentes 
(nanotecnología, biotecnología, tecnologías de la información y ciencias del conocimiento), áreas tecno-científicas en las que la región, en general, presenta capacidades de investigación exiguas, por lo que de no hacerse esfuerzos para superar esta situación, se agudizará el extractivismo.

Este incremento de la explotación de recursos agravará la degradación ambiental. Ya se comienzan a generar nuevos impactos, evidentes en la deforestación de selvas y bosques y la contaminación de cursos y reservorios de agua. Por ejemplo, la extracción del carbonato de litio requiere de gran cantidad de agua no salobre. Los salares que contienen las inmensa reservas del recurso se ubican en zonas desérticas, por lo que se está utilizando agua de acuíferos subterráneos prehistóricos, recurso que desde el punto de vista ecológico tiene un valor inestimable.

Se demuestra así que la estructura productiva de América Latina continúa anclada a las actividades inherentes al anterior paradigma tecno-económico. La consolidación global de los sistemas tecnológicos disruptivos colocará más presiones sobre sus recursos naturales, agudizando el fenómeno del "neoextractivismo". Si a ello se agrega una industria manufacturera que en la mayoría de los casos presenta limitadas capacidades tecnológicas (Westphal et al., 1985) y que pierde importancia en la composición del PIB, es casi inevitable el incremento de la brecha respecto a los países desarrollados y la agudización de las asimetrías. Por esta razón cabe preguntarse: ¿Compensan los costos, en términos de los impactos socioambientales y culturales negativos, de toda esta nueva fase del extractivismo? El mantenimiento del déficit social y la exclusión, los escasos avances tecnológicos y la degradación ambiental sugieren que no.

Es evidente que la crisis socioambiental y las profundas transformaciones tecnológicas colocan ingentes desafíos a la estructura tecnológica y científica de la región, y a su estructura productiva. En este escenario hay que revisar el papel que deben desempeñar las IES para abordar estos problemas y satisfacer sus requerimientos.

\section{Disrupciones tecnológicas y cuarta revolución industrial}

Gran parte de la investigación en tecno-ciencia que se realiza en las universidades en el ámbito global - especialmente en las áreas de química y ciencia de los materiales, matemáticas, ciencias de la computación e inge- 
niería - apuntan al desarrollo de tecnologías disruptivas (Thomsom Reuters, 2014a y b). De esta manera, estas instituciones se erigen en instancias clave para impulsar las transformaciones que actualmente experimentan diversos sistemas tecnológicos. Es tal el impacto de estas tecnologías que, aparte de modificar las concepciones mismas de producción, distribución y consumo, alterarán los hábitos cinestésicos de los seres humanos. ${ }^{5}$ La tabla 2 presenta las tecnologías que se considera tendrán mayores impactos socioeconómicos.

\section{Tabla 2 \\ Tecnologías disruptivas que impulsarán profundas transformaciones socio-técnicas}

\begin{tabular}{|l|l|}
\hline \multicolumn{1}{|c|}{ Área } & \multicolumn{1}{c|}{ Tecnología disruptiva } \\
\hline TIC & $\begin{array}{l}\text { Internet de las cosas } \\
\text { Cloud computing } \\
\text { Artefactos portátiles } \\
\text { Computación cuántica }\end{array}$ \\
\hline Nuevos materiales & $\begin{array}{l}\text { Nano materiales de grafeno } \\
\text { Nano materiales para electrodos de baterías }\end{array}$ \\
\hline Mecatrónica & Nueva generación de robots industriales \\
\hline Medicina & Próxima generación de secuenciación genómica \\
\hline Transporte & $\begin{array}{l}\text { Vehículos eléctricos } \\
\text { Vehículos auto-asistidos }\end{array}$ \\
\hline \multirow{2}{*}{ Almacenamiento avanzado de energía } & $\begin{array}{l}\text { Baterías de ion litio oxígeno } \\
\text { Baterías de ion sodio } \\
\text { Sistemas domésticos de almacenamiento }\end{array}$ \\
\hline
\end{tabular}

Fuente: el autor

Como puede apreciarse, inciden en los sistemas tecnológicos asociados prácticamente a todas las actividades socioeconómicas: producción industrial, energía y, prácticamente, en todos los servicios (transporte, finanzas, comunicaciones, etc.). Una característica importante de estas transformaciones es que no ocurren de manera independiente, sino mediante profundas in-

5 Sobre el uso de la tecnología, por ejemplo del automóvil, se avizoran modificaciones radicales: ¿Será necesario aprender a manejar un automóvil, entendiéndolo como el proceso de control del dispositivo y las reglamentaciones o se delegará estas habilidades a dispositivos autónomos? 
terrelaciones e interdependencias, lo que implica la emergencia de una revolución tecnológica (Pérez, 2002) o, probablemente, de varias revoluciones tecnológicas que están abriendo cauce a una cuarta revolución industrial.

Uno de los cambios más importantes en la estructura tecno-productiva se relaciona con la introducción de los sistemas "ciberfísicos" en la producción. Son redes de elementos interactivos que consideran sensores, máquinas, herramientas, sistemas de ensamblaje y partes, todas conectadas a través de redes de comunicación digitales (la nube), procesos que pueden ser controlados en forma remota. Algunos de sus componentes pueden actuar de manera independiente, estableciendo una diferencia fundamental con los tradicionales sistemas de control distribuido que, hasta ahora, constituyeron el núcleo de la automatización y control industrial (VDI, 2015). Pero surge una interrogante clave en la actual crisis socioambiental: ¿Incidirán todas estas transformaciones tecnológicas en el irracional consumo de recursos y la generación de contaminación? Algunas tecnologías disruptivas pueden contribuir a la disminución de GEI, atenuar la presión sobre los recursos naturales al posibilitar el diseño de procesos más eficientes y un aumento en el desarrollo de técnicas de reutilización y reciclaje. No obstante, la revisión de las tendencias en diversos sectores productivos y de servicios revela la consolidación de lo que se ha denominado gigantismo tecnológico (Mercado y Córdova, 2005), consustancial al paradigma económico del crecimiento continuo, que hace insignificantes los ahorros por mejoras en la eficiencia.

\section{Reelaboración de las formas de producir conocimientos}

La gravedad de los problemas socioambientales y la aparente imposibilidad de abordarlos desde el mainstream de la investigación y el desarrollo tecnológico, demandan una revisión de las formas de producir y usar el conocimiento. Así, paralelamente al imperativo desarrollo de la tecno-ciencia, está el papel que deben jugar otras formas de producción y transmisión de conocimientos (saberes ancestrales y/o tradicionales), capaces de impulsar formas alternativas de producción y consumo orientadas a satisfacer necesidades básicas de la población y, por lo tanto, más acordes con los postulados de la sustentabilidad. Además de sus posibilidades de ofrecer soluciones accesibles a los problemas y requerimientos de las comunidades. ¿Cuál debe ser el rol que deben desempeñar las universidades frente a estas formas 
alternativas de producción y uso del conocimiento? ¿Es posible el establecimiento de un diálogo de saberes que permita la conformación de espacios de co-creación de conocimientos?

Un caso concreto es el de las políticas de ciencia, tecnología y educación superior del Ecuador, que han establecido la necesidad de conjugar o al menos armonizar en la medida que sea posible, las tecnologías desarrolladas desde el mainstream de la tecno-ciencia con los saberes ancestrales, y entre ciencia y otros saberes. En el Plan de la Economía Social de los Conocimientos (PESC) (SENESCYT, 2017) se exploran posibles interrelaciones que apuntarían a lo que Vessuri (2004) destaca como la "hibridación de conocimientos".

Lo anterior abre cauces para una mayor interacción con la sociedad. El diálogo y el intercambio de saberes lleva a replantear los roles de la investigación en la educación superior. Del mismo modo, también exige redefinir algunos aspectos del papel de la universidad en desarrollo científico, tecnológico y económico, hasta retomar su lugar no solo como formadora individual, sino como ámbito de desarrollo de la cultura, la ciudadanía y la democracia (Zgaga, 2005). En otras palabras, se plantea una reelaboración de las formas de producir conocimientos.

\section{Ampliar prácticas de la investigación}

En la actualidad han surgido diversos movimientos de reelaboración de la producción de conocimiento. La "ciencia abierta" promueve una praxis científica que incluya el libre acceso a datos y publicaciones científicas, la posibilidad de compartir plataformas de investigación y desarrollar colaboraciones más amplias en proyectos científicos que pueden incluir la participación de profesionales (científicos) y practicantes no especializados (Barandiaran et al., 2015). Varios autores señalan que estas formas de organización y desarrollo de la investigación no implican la creación de un nuevo tipo de ciencia separada del sistema existente, sino la creación de una forma semi-institucionalizada que libere los resultados de la investigación. Aunque no se planteen explícitamente nuevos modos de generación de conocimiento, estas formas de organización pueden hacer más fluida su circulación, más allá de las comunidades de especialistas, posibilitando una mayor interacción entre estos y los practicantes no especializados. Indefec- 
tiblemente esto induce a una multidireccionalidad en el intercambio de información y conocimientos, por lo que dichos espacios pueden contribuir efectivamente a la hibridación de saberes (Hitner et al., 2017).

La "ciencia ciudadana" promueve el involucramiento activo de practicantes no profesionales en la investigación, considerando un amplio espectro de actividades que pueden ir desde proyectos realizados por pequeños grupos con intereses comunes, hasta proyectos internacionales que cuenten con la participación de instituciones de investigación sobre temas de interés de la sociedad (LERU, 2016). Los principales rasgos que configurarían esta praxis serían: la colaboración interdisciplinaria, la estructuración de redes de colaboración abierta ampliamente distribuidas y el estímulo a iniciativas que motiven a los ciudadanos a tener roles más activos en los proyectos (LERU, 2016).

Un tercer movimiento (la investigación participativa) va más allá, aportando métodos e instrumentos para una efectiva incorporación de las comunidades, basado en el establecimiento de relaciones mutuamente benéficas entre las universidades y los ciudadanos. Considera la co-creación y aplicación de conocimientos que incremente en ambos actores la capacidad para identificar temas y proponer soluciones. Se diferencia de la investigación tradicional en su propósito, procurando el beneficio de la comunidad, en una acepción amplia que puede considerar los ámbitos público, local, nacional y global (Stanton, 2012). También presenta diferencias importantes en los métodos, al prestar atención al carácter democrático que debe tener la colaboración, mediante la inclusión de todos los participantes en todas las fases de los proyectos: formulación, obtención y análisis de datos y aplicación de resultados. Esta última fase, aparte de suponer avances en el conocimiento, deberá también generar aportes que mejoren las condiciones de vida en las comunidades (Stanton, 2012).

$\mathrm{Al}$ adoptar estas prácticas no puede obviarse que la tecno-ciencia y otras formas de saber y conocer son incompatibles en su concepción y finalidades. Confrontar e integrar estas tendencias conlleva tensiones que es necesario considerar para una adecuada gestión de la producción del conocimiento. Por una parte, la imparable transformación socio-técnica global hace imperativo para la región el desarrollo de la tecno-ciencia, procurando orientarla hacia fines de mayor beneficio social y de soberanía. El fortalecimiento de la capacidad tecnológica de la industria, la agregación de valor a los recursos estratégicos aminorando los impactos socioambientales de su explotación, 
también son una prioridad. A la par, el desarrollo de vocaciones productivas locales que promuevan y rescaten conocimientos tradicionales y ancestrales que empoderen a las comunidades, constituyen espacios a construir mediante estas nuevas formas de organización y producción de conocimientos (por ejemplo: ciencia ciudadana, ciencia participativa).

Sin duda todos estos son grandes desafíos para las misiones de investigación y extensión. Pertinencia, vinculación con la sociedad e incremento de la participación, son temas pendientes en la agenda de la universidad latinoamericana.

\section{Redefinir la extensión}

El reposicionamiento de los temas de inclusión social y participación de los ciudadanos en la educación, ciencia y tecnología ha comenzado a recuperar la vigencia de la extensión universitaria, al ser esta misión la que permite el establecimiento de nexos entre las IES y otros actores de la sociedad con una mayor fluidez (Hitner et al., 2017).

Thiollent (1994), basado en experiencias en Brasil, propone que la extensión y la investigación se conciban como procesos de construcción social que cuenten con la participación de actores orientados al logro de objetivos concretos. En esta perspectiva, la metodología y los instrumentos de trabajo adquieren dimensiones participativas, críticas y reflexivas que favorecen propósitos de emancipación en los proyectos universitarios.

Se observa incluso el surgimiento de movimientos que hacen importantes cuestionamientos a la "renovada" tercera misión de la universidad (contribuir al desarrollo económico). Trencher (et al., 2014), basado en el análisis de experiencias en Norteamérica, Europa y Asia, apunta que la crisis socioambiental global está induciendo a muchas IES a desviar la atención del interés casi exclusivo hacia la generación de ingresos y el crecimiento económico, a constituirse en transformadoras sociales y co-creadoras. Esto desdibuja las fronteras entre los miembros de una comunidad (sociedad civil, gobiernos locales e industria) y los académicos, gracias a la instrumentación de mecanismos de colaboración orientados a la transformación física y sustentable de una localidad determinada para responder a requerimientos de algún grupo específico de la sociedad.

Aunque muchos de los métodos e instrumentos utilizados resultan ser prácticamente los mismos de los "paradigmas establecidos" — tales como 
la extensión agrícola, investigación y acción participativa, transferencia de tecnología y transdisciplinariedad - , lo novedoso radica en la emergencia de la función co-creativa, que consiste en la combinación de esos diversos modelos de acciones de compromiso social para responder a problemas socioambientales. Para ello es necesario internalizar los valores del desarrollo sustentable (Trencher et al., 2014).

Recientemente en EE.UU. un importante número de universidades ha realizado esfuerzos para revitalizar la participación cívica y comunitaria en sus localidades. En una tentativa que guarda similitud con las actividades de extensión latinoamericanas, subrayan la importancia de la participación de las comunidades en la orientación y desarrollo de sus funciones, haciendo uso de recursos institucionales para identificar y resolver los problemas que la comunidad afronta (Stanton, 2012). Considerando que el Modo 2 de generación de conocimiento plantea que su producción debe tomar en cuenta los contextos de aplicación, estas universidades proponen que parte del esfuerzo atienda a los problemas de las comunidades, lo que colocaría criterios que validarían el trabajo académico en sus espacios de localización (Stanton, 2012). Esto asigna importantes responsabilidades a las funciones de extensión, pues las universidades deberán constituir los puentes entre las comunidades y la segunda misión de las IES (la investigación), abriendo espacios a nuevas formas de desarrollar esta actividad (ciencia abierta, ciudadana o investigación participativa) bajo una perspectiva de pertinencia y relevancia.

El agravamiento de los problemas socioambientales y el imperativo de orientar las formas de vida por trayectorias más sustentables, parecen ser un punto de convergencia en el reenfoque de la extensión universitaria. Las IES, al ser factores claves en la participación ciudadana para enfocar estos problemas, proponer soluciones y participar en su resolución, tienen el gran desafío de repensar, en parte, sus otros grandes objetivos (formación e investigación) para renovar su contrato social y su vigencia.

\section{Conclusiones}

Un elemento renovador muy importante de la Reforma de Córdoba fue la valoración de las relaciones con la sociedad. Se planteó abrir la universidad mediante la proyección de la cultura y el asistencialismo, rasgos que conformaron y han caracterizado hasta hoy a su tercera misión: la extensión. 
La actual estructura tecno-económica global es, en gran medida, responsable de muchos de los graves problemas socioambientales que afronta el mundo y que colocan a un alto porcentaje de la población mundial en riesgo severo. Abordar temas como el calentamiento global, la contaminación y problemas sociales como la pobreza y la exclusión, es ineludible para las IES, pero para hacerlo de manera más efectiva deben asumir enfoques de investigación que trasciendan las formas tradicionales "intramuros", incorporando a través del diálogo a los saberes diversos y conocimientos de distintos actores sociales.

Simultáneamente, están ocurriendo grandes transformaciones tecnológicas que trastocan las concepciones establecidas de la producción y los servicios, con importantes consecuencias sobre la organización y división del trabajo, la estructura social e incluso sobre cada individuo, abriendo cauce a la cuarta revolución industrial. Pero al desarrollarse en la lógica del crecimiento continuo no plantean alternativas al actual patrón de desarrollo.

Esto coloca un doble desafío a las IES latinoamericanas. Responder, por una parte, al impuesto por las citadas transformaciones tecnológicas, partiendo de un rezago notable de su estructura productiva, basada en actividades primarias y de explotación de recursos naturales, con severas consecuencias ambientales. Ello mediante el desarrollo de una tecno-ciencia orientada hacia un mayor beneficio social y de soberanía. Por la otra, a las demandas de participación e inclusión que llevarían implícitas la reelaboración de las formas de producción de conocimiento.

Todo esto plantea redefinir la extensión, al constituir esta el espacio inmejorable de interacción con la sociedad. Aparte de la proyección cultural y las labores asistenciales, la universidad deberá desarrollar los mecanismos de correaje para la transmisión e intercambio de información y conocimientos entre las comunidades y los investigadores, con el fin de posibilitar nuevas agendas de proyectos y contribuir a crear los espacios que permitan la implantación de las formas alternativas para realizar la actividad (ciencia abierta, ciudadana o investigación participativa). En esta medida, se transforma también la segunda misión de la universidad.

\section{Bibliografía}

Barandiaran, X. Araya, D. y Vila-Viñas, D. (2015). Ciencia: investigación colaborativa, participativa y abierta. En Buen Conocer/FLOK Society: modelos 
sostenibles y políticas públicas para una economía social del conocimiento común y abierto en el Ecuador. Quito: IAEN.

Bruckmann, M. (2015). El litio y la geopolítica de integración de América Latina. En F. Nacif, y M. Lacabana (eds.), ABC del litio sudamericano soberanía, ambiente, tecnología e industria. Quilmes: Centro Cultural de la Cooperación Floreal Gorini/Universidad Nacional de Quilmes.

Bussines Europe. (2014). Revisión intermedia garantizar resultados. Recuperado de https://bit.ly/2OLz4lA/

Fresán Orozco, M. (2004). La extensión universitaria y la universidad pública. Reencuentro, 39, 47-54.

Geyer, R. Jambeck, J. y Lavender Law, K. (2017). Production, use, and fate of all plastics ever made. Science Advance. Recuperado de https://bit. ly/2Mw1RwL/

Hitner, V., Carlotto, M. y Mercado, A. (2017). Agenda 2035 para la educación superior en el Ecuador. Quito: SENESCYT.

Hope, K. (16 de enero de 2017). Eight billionaires “as rich as world's poorest half". BBC News. Davos: BBC. Recuperado de https://bbc.in/2vTiRDM/

Hughes, T. 1987 "The evolution of large technological systems" en Bijker, W.E.,Hughes, T.P. and Pinch, T.J. (eds) 1987 The Social Construction of Technological Systems (Cambridge: MIT Press).

LEAGUE OF EUROPEAN RESEARCH UNIVERSITIES (LERU) (2016). Citizen science at universities: Trends, guidelines and recommendations. en: http:// www.uib.cat/digitalAssets/409/409499_leru_ap20_citizen_science.pdf

Mercado, A. y Córdova, K. (2005). Desarrollo sustentable-industria: más controversias menos respuestas. Ambiente \& Sociedad, 8(1).

SENESCYT (2017). "Plan de Economía Social de los Conocimientos, la Creatividad, la Innovación y los Saberes Ancestrales”. En: http://observasur.org/ wp-content/uploads/2017/06/Diagrama-PESC-Revisado.pdf

Salleh, A. (13 de febrero de 2015). Plastic ocean: study names worst polluters. $A B C$ Science. Recuperado de https://ab.co/2Bwvryb/

Serna Alcántara, A. (2007). Misión social y modelos de extensión universitaria: del entusiasmo al desdén. Revista Iberoamericana de Educación, 43(3).

Stanton, T. (2012). New Times Demand New Scholarship II: Research Universities and Civic Engagement: Opportunities and Challenges. Journal of Higher Education Outreach and Engagement, 16(4), 27.

Thiollent, M. (1994). A inserção da pesquisa-ação no contexto da extensão universitária. Recuperado de https://bit.ly/2wcob4B/ 
Thomsom Reuters (2014b). Research Fronts 2014. Recuperado de https://bit. ly/2MSVFvH/

Thomsom Reuters. (2014a). Research Fronts 2014: 100 Top Ranked Specialties in the Sciences and Social Sciences. Recuperado de https://bit.ly/2MSbbrJ/

Trencher, G. Yarime, M. McCormick, K. Doll, C. Kraines, S (2014). Beyond the Third Mission: Exploring the Emerging University Function of Co-creation for Sustainability. Science and Public Policy, 41(2), 151-179.

Tünnermann, C. (ed.) (2008). Noventa años de la Reforma Universitaria de Córdoba: 1918-2008. Buenos Aires: CLACSO.

VDI. (2015). Industry 4.0 A Discussion of Qualifications and Skills in the Factory of the Future: A German and American Perspective. Recuperado de https:// bit.ly/2OK2PD5/

Vessuri, H. (2004). La hibridización del conocimiento: la tecnociencia y los conocimientos locales a la búsqueda del desarrollo sustentable. Convergencia, revista de ciencias sociales, 11(35), 171-191.

WB. (2018). The Changing Wealth of Nations 2018 Building a Sustainable Future. Recuperado de https://bit.ly/2FW7D7B/

Westphal, L., Kim, L. y Dahlman, C. (1985). Reflections on the republic of Korea's acquisition of technological capability. En N. Rosemberg y C. Frischtak (eds.), International Transfer of technology: Concepts, Measures, and Comparisons. NY: Praeger Press.

Williams, G. (2016). Higher education: Public good or private commodity? London Review of Education, 14(1).

WMO. (2017). Wmo Greenhouse Gas Bulletin. Recuperado de https://bit. ly/2A0py $7 \mathrm{~h} /$

WMO. (18 de enero de 2018). WMO confirms 2017 among the three warmest years on record. Recuperado de https://bit.ly/2mV8YRi/

Zgaga, P. (2005) Higher education for a democratic culture - The public responsibility. En: The public responsibility for higher education and research. Weber, L. Bergan, S (eds). Council of Europe Publishing. Disponible en: https://goo.gl/32nb7o 